\title{
INTEGRATING TECHNOLOGIES FOR DEMAND RESPONSE IN BLOCKS OF BUILDINGS - A UK CASE STUDY
}

\author{
Tracey Crosbie ${ }^{1}$, John Broderick ${ }^{2}$, Muneeb Dawood ${ }^{3}$, Richard Charlesworth ${ }^{4}$, Vladimir \\ Vukovic $^{5}$, Michael Short ${ }^{6}$, and Nashwan Dawood ${ }^{7}$
}

\begin{abstract}
Flexibility in contemporary energy systems is predominantly delivered by fossil fuels. Low carbon energy services are required to avoid dangerous climate change, however, in the electricity sector, energy flows must be balanced instantaneously, and many renewable resources are either variable, uncertain or both. Demand Response (DR) enables consumers to play a significant role in the delivery of flexibility on the electric grid by reducing or shifting their electricity usage during periods of stress or constraint. The value of DR to blocks of buildings depends on the telemetry and control technologies in existing building management systems and the potential revenue sources. To encourage the growth of DR services' and reap the potential benefits, it is necessary to characterise the economic and environmental benefits of DR. The EU Horizon 2020 co-funded project "Demand Response in Blocks of Buildings" (DR-BOB: www.dr-bob.eu) aims to do just that. This paper describes the technical approach taken by the DR-BOB project at its Teesside University site, focussing on the challenges encountered and the solutions proposed for this city centre campus. It updates previous work (Crosbie et al, 2016) that has described the broader principles and technologies being evaluated at four sites across Europe.
\end{abstract}

Keywords: demand response (DR), flexibility, smart grid, electricity networks, blocks of buildings.

\section{INTRODUCTION}

The majority of the flexibility in contemporary energy systems, the ability to satisfy demand for energy services at any point in time, is delivered by fossil fuels. However, the necessity of climate change mitigation (IPCC 2014) means that alternative, low carbon means of delivering this flexibility are required. Furthermore, many renewable resources are either variable, uncertain or both, placing an increased burden on other parts of the energy system. This problem is particularly acute in the electricity sector, where energy flows must be balanced instantaneously, and storage and inertia are constrained by the physical properties of the system.

Demand response (DR) provides an opportunity for consumers to play a significant role in the delivery of flexibility on the electric grid by reducing or shifting their electricity usage during periods of stress or constraint. This is typically rewarded with some form of financial incentive as a direct payment for a provided services, or in some cases a penalty

\footnotetext{
Senior Research Lecturer, SSE, Teesside University, Middlesbrough, TS1 3BX, t.crosbie@tees.ac.uk Research Associate, SSE, Teesside University, TS1 3BX, j.broderick@tees.ac.uk Research Associate, SSE, Teesside University, TS1 3BX, m.dawood@tees.ac.uk Siemens UK, Fairburn House, Green Lane, Leeds, LS25 2AF, richard.charlesworth@siemens.com Senior Research Lecturer, SSE, Teesside University, Middlesbrough, TS1 3BX, v.vukovic@tees.ac.uk Reader, SSE, Teesside University, Middlesbrough, TS1 3BX

Professor, Director Technology Futures Institute, Teesside University, n.dawood@tees.ac.uk
} 
for non-participation. DR is recognised as being beneficial for customers and energy networks (SEDC 2013), specifically by reducing the cost of increased penetration of renewable energy, easing capacity constraints on distribution networks, reducing generator margin requirement and the costs of traditional spinning reserve, and increased efficiency of asset utilisation.

The potential for "blocks of buildings", to participate collectively in DR has been given preliminary investigation by academic researchers (Crosbie et al. 2015), however, commercial DR implementations aimed at small or medium scale customers have failed to meet expectations (Olivares et al. 2015). Significantly, the value of DR to blocks of buildings depends on the telemetry and control technologies embedded in existing building management systems and the potential revenue sources, both of which vary according to specific local and national circumstances (SEDC 2013). The EU Horizon 2020 co-funded project "Demand Response in Blocks of Buildings" (DR-BOB, www.dr-bob.eu) aims to clarify just that. This contribution describes the technical approach taken by the project at its Teesside University site, specifically focussing on the challenges encountered and the solutions proposed for this city centre campus. It updates previous work (Crosbie et al. 2016) that has described the broader principles and technologies involved in the project across all four sites.

\section{ICT FOR DEMAND RESPONSE}

\subsection{The Integration Opportunity}

There is a lack of integrated tools supporting optimisation, planning and control/management of demand side equipment (Olivares et al. 2015). As a result, previous demand response implementations aimed at small or medium scale customers have failed to meet their expected potential. Particular technical deficiencies have been identified as:

1. Out-dated metering technologies preventing relevant real-time information reaching customers;

2. Undue complexity in the presentation of information for user engagement;

3. Absence of means for customers to respond to real-time prices and demand signals; Furthermore, without scalable integrated tools supporting optimisation, planning and control of demand side equipment there is a perpetuation of the general assumption of demand inelasticity (Kim et al. 2011).

The assembly of existing technologies, software components and concepts into a scalable, low cost and open platform for supply/demand optimisation is the key technical innovation in the DR-BOB project. Therefore, the innovation in the project lies, not in the development of new technologies but rather in the integration of existing technologies and their application at the level of blocks of buildings. For the purposes of the DR-BOB project, a "block of buildings" is defined as i) a group of 3 or more real, physical, buildings, ii) sharing a common energy governance, iii) used the whole year or during workdays by multiple schedules of occupancy, and iv) served by the same Distribution Network Operator (DNO). In total, the DR-BOB solution will be demonstrated at four European sites with different ownership structures, functions, infrastructures, climates, and regulatory environments. This paper focuses on the system deployed at one of those sites. 
Crosbie, T., Broderick, J., Dawood, M., Charlesworth, R., Vukovic, V., Short, M., and Dawood, N.

\subsection{UK Pilot Site}

Teesside University, Middleborough, UK was selected as a pilot site for the demonstration of technologies. The main rationale is that the BMS to DR integrating technology, the Local Energy Manager (LEM) is being developed by the academic team at Teesside University.

Teesside University has a town centre location at the mouth of the River Tees in North East England. Its main purpose is to facilitate teaching and research activities. Approximately 20,000 students and 2,500 staff work and study in the campus buildings which house offices, technical laboratories, computer clusters and lecture theatres. In total the campus has 33 buildings, 5 of which provide residential accommodation for students.

We have identified a block of buildings, connected to a common BMS system featuring assets which could participate in demand response through a combination of physical trials and simulation. Key assets within the buildings are:

- Approximately 400kWe BMS controllable HVAC (chillers, AHU, split systems).

- A CHP plant (230 kWe, $358 \mathrm{kWth}$ ) used as a local energy production system for the site contributing to part electrical and thermal demand. Heat can be rejected during summer months to maintain electrical generation if required.

- A district heating system fed by the CHP and two other gas boilers.

- A 176kW backup generator (diesel-fired) and Uninterruptible Power Supply (UPS) protecting campus servers.

\subsection{Technical elements of the DR-BOB solution}

The key functionality of the DR-BOB solution is the coordination of energy production, consumption and storage with demand response incentives. The solution will be operated by the Estates department within the organisation, allowing for opting of assets into and out of DR programmes with optimisation adjusted to either maximise economic return or minimise $\mathrm{CO} 2$ emissions. The $\mathrm{DR}-\mathrm{BOB}$ solution provides a scalable cloud based central management system, supported by a local real-time energy management integrating the following three tools and technologies:

- Siemens DEMS® (Siemens 2017)

- Local Energy Manager (LEM) developed by Teesside University during the IDEAS project (Short et al. 2015)

- Consumer Portal - developed by GridPocket

In summary, DEMS provides macro-level energy management and control, interfacing with network operator (TSO and/or DNO/DSO) DR programmes. The LEM provides micro level optimised energy management and building interfaces, while the Customer Portal offers a user interface for energy management and building occupant engagement.

An overview of the ICT infrastructure for UK site is given in Figure 1 with a description of the functionality of the elements following.

\subsubsection{Metering system}

Energy meters are associated with buildings and specific electrical and heat loads and generation across the site, including CHP plant, chillers, air handling units, boilers etc. Meter readings are sent to data loggers in real-time, which in turn send readings over a $3 \mathrm{G} / 4 \mathrm{G}$ network to a third party metering services company, subsequently uploaded to a FTPS server located on the Gateway PC every 15 minutes. 


\subsubsection{Gateway PC}

For stability and security of the Teesside University local campus IP network, it was decided to avoid direct IP connectivity between the DEMS external IP network and the TU local campus IP network. A Gateway PC is used to act as a host and enable information exchange between IT systems located on the TU local campus IP network (BMS, metering FTP server) and systems located on external IP network (LEM, DEMS). The Gateway PC is connected to the Teesside University IP network and interfaces to the LEM to send meter readings using a high-speed TIA-232 serial link.

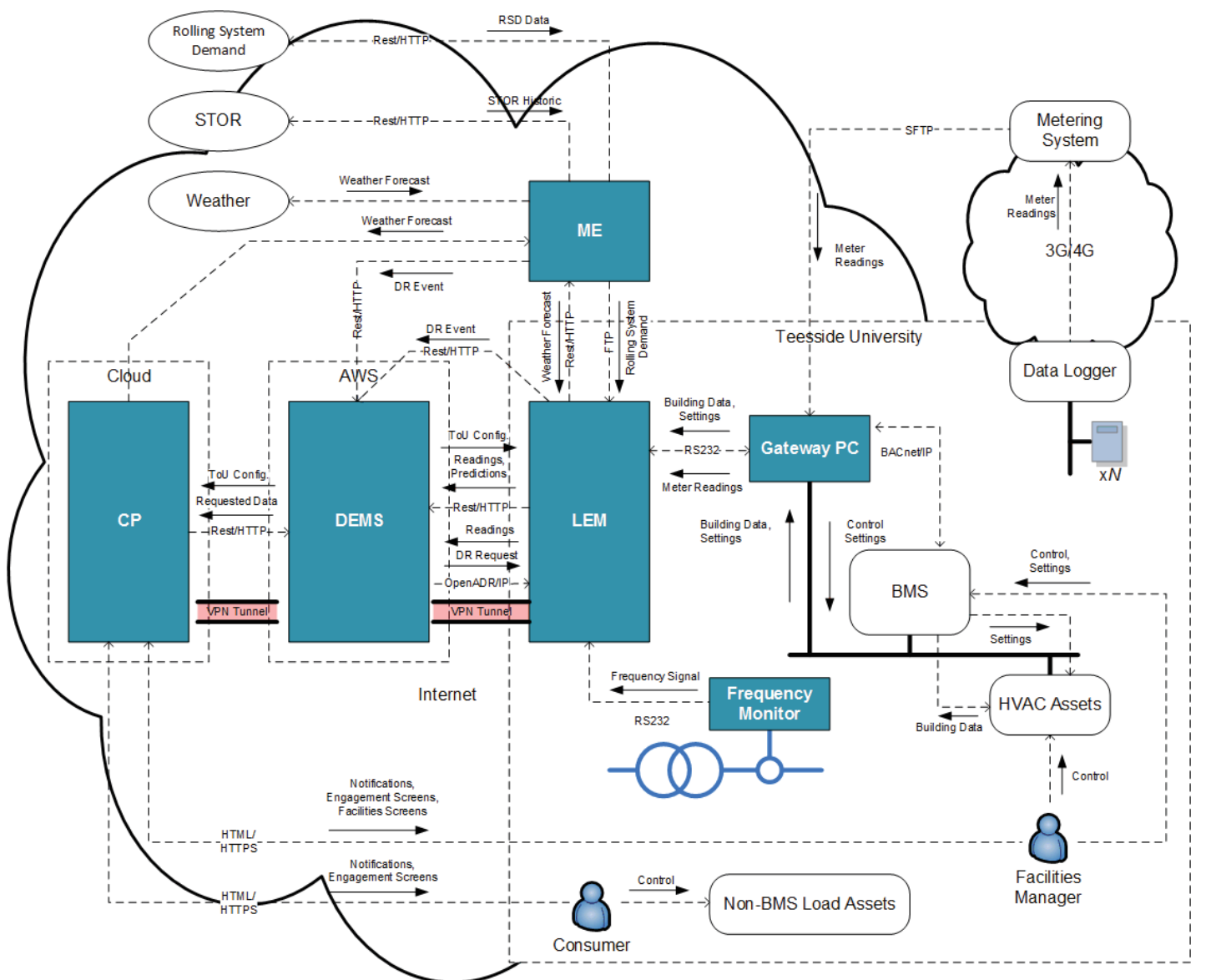

Figure 1: UK Site Architecture, for key see following section.

\subsubsection{BMS system}

The Schneider StruxureWare BMS installed in the demonstration buildings operates in a client-server configuration. The BMS server application hosts a database to store BMS settings and communicates with Satchwell Sigma remote terminal controllers associated with various assets. Communication with the LEM is conducted over BACnet/IP via a Gateway PC to retrieve asset information (HVAC status, room temperatures, flow/return temperatures) and introduce new set point schedules in response to DR events. Such communication was not possible with the legacy BMS configuration and the upgrade required substantial investment. A "LEM Slave" alternative, an Arduino based interface with wireless TIA-485 capability associated with a BMS outstation, was investigated, however, this would have had much reduced functionality. 
Crosbie, T., Broderick, J., Dawood, M., Charlesworth, R., Vukovic, V., Short, M., and Dawood, N.

\subsubsection{Market Emulator (ME)}

In the DR-BOB trial there are no explicit (c.f. implicit, e.g. time of use tariff) DR contracts with an energy utility company with which to generate DR events. A software entity called a Market Emulator has been developed as a substitute for this purpose, generating and passing events on to DEMS using the HTTP protocol. The ME collects National Grid consumption data in real-time to generate TRIAD warnings, and uses historic STOR and DTU data to generate realistic DR event requests. The ME also gathers weather forecasts from a web source (http://api.met.no/weatherapi/) and retrieves relevant energy market data for each site for processing by the LEM and subsequent evaluation. The ME uses a REST-based API to retrieve energy market data and weather forecast data.

\subsubsection{DEMS}

DEMS sits at the centre of the DR-BOB DR energy management solution; its role is to manage the balancing of supply and demand through manipulation of connected assets via the building management system across a number of buildings and sites. It is the central point for the initiation of DR events and will manage the requests for generation, demand or storage to satisfy the request.

DEMS can be configured to support multiple types of DR programmes providing both emergency and economic dispatch. DEMS can interface with residential, commercial, or industrial sites to provide more flexibility in how Energy Supply Companies (ESCOs) create their DR programmes. DEMS also provides the ability to define workflow processes so that DR events are managed according to utility business processes. DEMS is hosted in the cloud and in the DR-BOB project receives DR events generated by the Market Emulator (ME) via HTTP protocol. DEMS store these DR events in a database, generated manually or in an automated fashion based on messages received from the ME. These DR events can be retrieved by LEM in OpenADR format.

\subsubsection{Local Energy Manager (LEM)}

The Local Energy Manager (LEM) avoids the requirement for DEMS to have to scale to meet the real-time energy management needs of potentially many buildings. Handing this responsibility to site specific LEMs will improve reliability and reduce costs because the communications and centralised computing will be kept to a minimum.

Building management has come a long way since its first introduction, with the methods used to control HVAC, lighting and other energy consuming equipment becoming significantly more sophisticated. However, some BMS solutions lack the capability to manage the addition of generation and storage assets. The LEM plays an important, complementary role by providing these optimisation and interface capabilities. The LEM software is hosted on a rack-mounted industrial server RuggedCom RX1400 and equipped with heat and electrical load prediction and commitment/dispatch optimisation software developed previously in the IDEAS project.

Where assets are not directly connected to the BMS - which is often the case with embedded generation - the LEM is able to integrate directly. By taking this local view the LEM will be able to take the responsibility for the decisions on what load, storage or generation it has to spare, working proactively and on-demand to prepare for and manage DR events. Following the occurrence of a specific DR event in the local market, the LEM will also resolve the local commitment/dispatch optimisation problem. An adaptive autoregressive model relating the key parameters of the building and control environments is automatically updated by the LEM software with each new measurement that is taken. This model is then employed in an optimizer to minimise energy consumption at specified 
times (DR events) ensuring occupant comfort constraints are met as closely as possible. This is a form of Model Predictive Control (MPC) which is known to be effective in smartgrid applications (Li et al, 2013). The LEM is also equipped with a high-accuracy Arduino microcontroller based frequency monitor, which samples the local electrical grid frequency at $1 \mathrm{~Hz}$ and communicates this information to the LEM over TIA-232. This allows the LEM to participate not only in traditional explicit DR but emerging forms of DR based upon decentralized frequency regulation concepts. We will assess the ability of the DR-BOB solution to dispatch assets promptly and pro-actively for participation in frequency response programmes.

In addition to reducing computational demands upon the DEMS, the proposed integration with the LEM will increase security, as detailed internal operational information related to a building does not need to leave the domain managed by LEM. The optimisation embedded in the LEM will build on work conducted as part of the IDEAS project. As with many technology evolutions, the components that go to make up the whole are kept separate to prove their capabilities before being combined to reduce costs in the production process and for the end user. Following this philosophy, it is perfectly conceivable that the LEM functionality could be embedded in a BMS in future.

\subsubsection{Connectivity}

LEM and DEMS connectivity is via a secure VPN tunnel using OpenVPN technology. The benefit of using OpenVPN is its simple setup on the server and client side. LEM sends meter readings and meter reading forecasts to DEMS in UAAV2 XML format using SFTP for the transfer. LEM polls for, and receive DR events from DEMS in OpenADR format. LEM also receives weather forecast from an external source, in this case the ME using a web based HTTP protocol.

\subsubsection{Customer Portal}

The Consumer Portal is multi-device friendly application based on a unified platform for all web browsers, including tactile web terminals (GridPocket, n.d.). It has several customisable features and extendable widgets and supports multiple languages. Essentially it will provide an information and control interface for the DR-BOB solution, including, periodic consumption graphics, real-time energy analysis, historical performance in DR events, energy costs and incentives. It offers the site facility manager to receive notifications and the ability to opt assets into and out of DR programmes. It provides DR events and notifications on a public display for building occupants to manually control load assets, such as IT and laboratory equipment.

\subsubsection{Systems configuration}

In combination, DR-BOB DR solution technologies will provide open connectivity to both SCADA/utility communications and customer side advanced metering infrastructures. The decentralised approach - allowing both supply side and DR to be hierarchically optimised between blocks of buildings and other infrastructures, with automatic distribution of results via building management systems - removes some of the burden and alleviates the complexities involved in individual customer or resident participation. The advantage of Siemens products in this solution is their support for standards such as IEC61879-5-104 and OpenADR, and flexible architecture that will enable new adaptors to be added to support new standards in the future. 


\section{DEMONSTRATION OF THE DR-BOB SOLUTION}

The DR-BOB solution is being deployed at the Teesside University site in early 2017 with trials scheduled to start in September 2017 for 12 months. This will gather data for evaluation of the technology and its operation at this site and others. Key Performance Indicators include reductions in energy and electricity demand, reductions in peak-power to minimum demand, and an assessment of the Return on Investment (ROI).

Development of the solution to date has identified a number of general limitations of DR in commercial buildings:

1. The bulk of electrical demand in commercial buildings may not be able to be time shifted due to the pervasive use of IT equipment in the service sector.

2. There is difficulty in identifying major dispatchable loads for control; boiler plate power ratings may not correspond to high energy consumption per se, nor high demand at the time of DR requests.

3. DR revenues are currently low value in proportion to i) overall operational expenditure and even energy expenditure alone, and ii) complexities of establishing DR within existing infrastructure and organisational practices.

Specific technical challenges associated with the TU site have also become apparent:

1. There is limited day to day flexibility in timing educational activity due to the need for planned timetables. Further, the contemporary neo-liberal education environment introduces new sensitivities around "the student experience" in light of the high fees charged for tuition.

2. The specific optimisation algorithms are unproven in this context. Notably, the heterogeneous spatial and temporal concentration of occupants encountered in an education institution may introduce new problems.

3. Existing metering infrastructure has presented difficulties, specifically due to i) the limited extent of sub-metering, ii) the multiplicity of systems handling metering data, iii) data dropouts due to data logger, transmission or server failure, iv) relatively low frequency of monitoring (half hourly), and v) high latency (24 hours) of existing infrastructure. Such difficulties may be expected in further deployment.

4. Need for consensus between multiple actors involved in energy management, each responsible for operation of different demand response assets, e.g. IT system operation is primary responsibility of IT services department, student housing administration is in charge of student accommodation units, while estates department is in charge of overall energy management.

\section{CONCLUSIONS}

The individual components required for the DR-BOB DR energy management solution are already in existence and in some cases mature but have required refinement and integration for simultaneous deployment. This paper has described their configuration at Teesside University, using open communication architectures, protocols and standards. The integration afforded by the LEM, DEMS and Consumer Portal technologies addresses technical deficiencies in energy meter data display and building control that have prevented DR flourishing in commercial buildings. The effectiveness and reliability of these real-time systems will be demonstrated and evaluated in operational situations from 
March 2017 until February 2019. Through a substantial dissemination programme we will also investigate the commercial scalability of the proposed solution; the interfaces and algorithms applicable to the four demo sites may not necessarily be representative of major market opportunities.

\section{ACKNOWLEDGMENTS}

This work is being performed as part of the DR-BOB project $(01 / 03 / 16-28 / 02 / 19)$ which is co-funded by the EU's Horizon 2020 framework programme for research and innovation under grant agreement No 696114. The authors wish to acknowledge the European Commission for their support. The authors also gratefully acknowledge the contribution of the DR-BOB project partners to the work presented.

\section{REFERENCES}

Crosbie, T., Dawood, M., Short, M., Brassier, P., Dorcome R., Huovila, A. and Ala-Juusela, M. (2015) IDEAS Project Deliverable D2.3, Generalised business models. Available at: https://www.researchgate.net/publication/287948614 Generalised business models

Crosbie, T., Vukovic, V., Short, M., Dawood, N. N., Charlesworth, R., Brodrick, P. (2016) 'Future demand response services for blocks of buildings' in Hu, J., Leung, V.C.M., Yang, K., Zhang, Y., Gao, J., Yang, S. (Eds.) Smart Grid Inspired Future Technologies: Revised Selected Papers from the First International Conference, SmartGIFT 2016, Liverpool, UK, May 19-20, 2016, http://www.springer.com/us/book/9783319477282

GridPocket (n.d.) EcoTroksTM energy efficiency. Available at: http://www.gridpocket.com/products/behavioral-energy-efficiency-program/

IPCC (2014) Climate Change 2014: Synthesis Report. Contribution of Working Groups I, II and III to the Fifth Assessment Report of the Intergovernmental Panel on Climate Change [Core Writing Team, R.K. Pachauri and L.A. Meyer (eds.)]. IPCC, Geneva, Switzerland, $151 \mathrm{pp}$.

Kim, J.H. and Shcherbakova, A. (2011) Common failures of demand response, Energy, Vol. 36, pp. 873-880, http://dx.doi.org/10.1016/j.energy.2010.12.027

Li, M. and Luh, P.B. (2013) A Decentralized Framework of Unit Commitment for Future Power Markets, IEEE Power \& Energy Society General Meeting

Olivares, O., Mehrizi-Sani, A., Etemadi, A.H., Cañizares, A.H., Iravani, R. Kazerani, M., Hajimiragha, A.H., Gomis-Bellmunt, O., Saeedifard, M., Palma-Behnke, R., JiménezEstévez, G. A., Hatziargyriou, N.D. (2014) Trends in Microgrid Control, IEEE Transactions on Smart Grid, Vol. 5, No. 4.

SEDC (2013) Smart Energy Demand Coalition: A Demand Response Action Plan for Europe. Available at: http://smartenergydemand.eu/wpcontent/uploads/2013/06/SEDC-DR-FINAL-.pdf

Short, M., Dawood, M., Gras,D., et. al. (2015) IDEAS Project Deliverable D4.1: A prototype neighbourhood energy management tool

Siemens (2017) DEMS: Decentralized Energy Management. Accessible at: https://w3.siemens.com/smartgrid/global/en/resourcecenter/emeterresources/Documents/DEMS_DataSheet.pdf 